\title{
A sampler for atmospheric volatile organic compounds by copter unmanned aerial vehicles
}

Karena A. McKinney (1,2) (*), Daniel Wang (2), Jianhuai Ye (2), Jean-Baptiste de Fouchier (2),

Patricia C. Guimarães $(3,4)$, Carla E. Batista $(3,4)$, Rodrigo A. F. Souza $(3,4)$, Eliane G. Alves

$(3,5)$, Dasa Gu (6), Alex B. Guenther (6), Scot T. Martin (2,7) (*)

\section{Supplementary Material}

\section{Description of the calibration standard.}

The thermal desorption cartridge samples were calibrated using a commercial standard from Apel-Riemer Environmental Inc. on a daily basis. The standard contained isoprene, $\alpha$-pinene, $\beta$ pinene, limonene, myrcene, aromadendrene, methyl vinyl ketone, terpinolene, methacrolein, farnesol, cis-3-hexenyl-acetate, caryophyllene, 3-carene, longifolene, acetaldehyde, methanol, acetone, benzene, toluene, o-xylene, 1,3,5-trimethylbenezene, 1,2,4-trichlorobenzene, 1,3,5triisopropylbenzene, $\mathrm{C}_{3}-\mathrm{C}_{10}$ alkanes, and $\mathrm{C}_{3}-\mathrm{C}_{6}$ alkenes.

Table T1. Sampler Components.

\begin{tabular}{llll}
\hline Item & Manufacturer & Part number & Specification \\
\hline Pressure sensor & NXP & MPX4100AP-ND & 20 to 105 kPa $\pm 1.8 \%$ FS \\
Valve manifold & NResearch Inc. & $161 \mathrm{~T} 102$ & 5 sample channels \\
Valve driver board & NResearch Inc. & 161D5X24 & \\
Flow sensor & Omron & D6F-P & 0 to 1 SLPM $\pm 5 \%$ FS \\
Micro Diaphragm pump & Parker Hargraves & E155-11-050 & 0.1 to 0.6 L min ${ }^{-1}$ \\
Needle Valve & Universal Power Conv. & F-2822-51-B85-K-V & \\
Microcontroller & Arduino & Uno & \\
Power & DJI Matrice 600 & TB48S & 18 VDC, 130 Wh, 2.5 Wh used \\
\hline
\end{tabular}




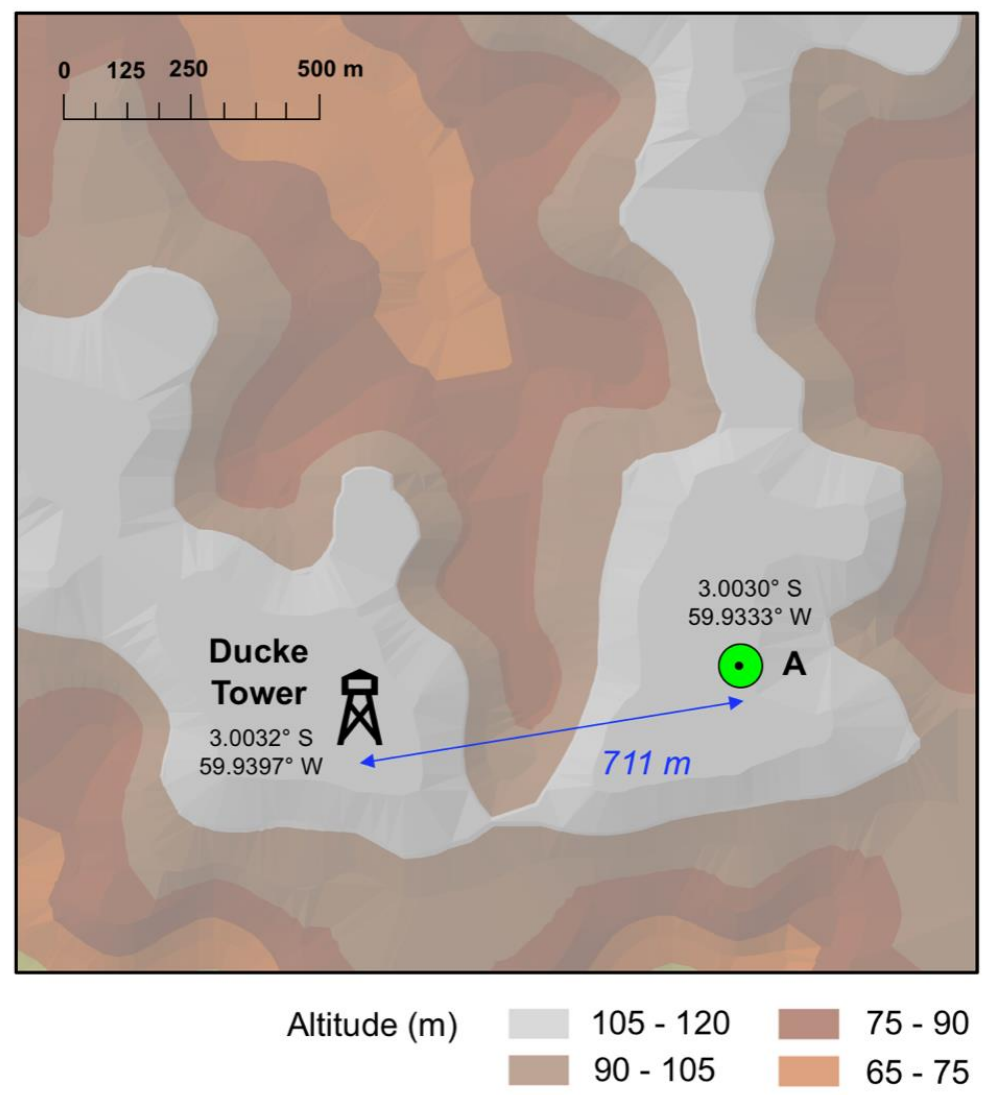

Figure S1. Topographical map of the experimental site. Labels indicate the location of the tower and point $\mathrm{A}$, where samples were taken with the UAV. 